\title{
Multi-messenger real-time follow-up of transient events with ANTARES neutrino telescope
}

\section{Dornic*}

Aix Marseille Univ, CNRS/IN2P3, CPPM, Marseille, France

E-mail:dornicecppm.in2p3.fr

\section{A. Coleiro}

APC, Univ Paris Diderot, CNRS/IN2P3, CEA/Irfu, Obs de Paris, Sorbonne Paris Cité, France

IFIC - Instituto de Física Corpuscular (CSIC - Universitat de València) c/ Catedrático José Beltrán, 2 E-46980 Paterna

Valencia, Spain

E-mail: alexis.coleirodific.uv.es

on behalf of the ANTARES Collaboration

By constantly monitoring at least one complete hemisphere of the sky, neutrino telescopes are well designed to detect neutrinos emitted by transient astrophysical events. Real-time searches for ANTARES neutrino candidates coincident with gamma-ray bursts, High-Energy Starting Events and Extremely High-Energy Events detected by IceCube and gravitational wave (GW) candidates observed by LIGO/Virgo are performed. By requiring coincident detection, this approach increases the sensitivity of the telescope and the significance of a potential discovery. The latest results of these analyses will be presented. In particular, a neutrino follow-up is performed after the detection of GW events by the LIGO/Virgo collaboration. Because of the good angular accuracy of neutrino telescopes compared to current GW detectors with two interferometers, a coincident detection would drastically constrain the position of the GW source on the sky, bringing valuable information for subsequent electromagnetic follow-ups. Since no coincident ANTARES event has been detected so far, the neutrino fluence and the total energy emitted in neutrinos are constrained for each GW alert.

35th International Cosmic Ray Conference - ICRC2017

10-20 July, 2017

Bexco, Busan, Korea

${ }^{*}$ Speaker. 


\section{Introduction}

Time-domain astroparticle physics has entered an exciting period with the recent development of wide-field-of-view instruments, communication strategies and low latency alert triggering of gravitational wave and high-energy neutrino (HEN) signals, but also across the electromagnetic spectrum. In particular, neutrinos represent unique probes to study high-energy cosmic sources. They are neutral, stable and weakly interacting. Contrary to cosmic rays (CRs), they are not deflected by the magnetic fields and unlike high-energy photons, they are not absorbed by pair production via $\gamma \gamma$ interactions with cosmic microwave and infrared backgrounds. A HEN diffuse flux of cosmic origin has been identified by the IceCube telescope (see e.g. [1]), the sources of which have still to be identified. In this context, multi-messenger approaches consisting in simultaneously looking for the same sources with both neutrino telescopes, gravitational wave interferometers and/or multi-wavelength facilities can constitute a viable mean of locating HEN/CR sources and thus further understanding the acceleration mechanisms at play in these sources.

Search for transient sources of HEN is promising since the short timescale of emission drastically reduces the background level, mainly composed of atmospheric muons and neutrinos and consequently increases the sensitivity and discovery potential of neutrino telescopes. In particular, specific strategies are developed to look for neutrino events in both time and space coincidence with transient events announced by public alerts distributed through the Gamma-ray Coordinated Network (GCN) or by private alerts transmitted via special channels. Offline analyses are performed to search for neutrino counterparts to catalogued flaring sources and will be presented at this conference [2]. Hereafter, we describe the real-time follow-up analyses performed with ANTARES after the detection of transient events which require low latency follow-up: gravitational wave events by LIGO/Virgo (Section 3), high-energy neutrino candidates by IceCube (Section 4) and Gamma-Ray Bursts and Fast Radio Bursts (Section 5).

\section{Data acquisition and real-time alert reception in ANTARES}

The data acquisition system of the ANTARES detector is based on the "all-data-to shore" concept [3]. The time and charge amplitude of all the photomultiplier (PMT) signals above a threshold of 0.3 photoelectrons are sent to a computing farm onshore for processing. A filter is applied on shore to select the physics events among the raw dataset structured in time slices of $104.85 \mathrm{~ms}$ and dominated by hits due to the optical background produced by bioluminescence and radioactive decay of ${ }^{40} \mathrm{~K}$. Two filtering algorithms look for a combination of local clusters of hits within a time winow of $2.2 \mu \mathrm{s}$. The first one selects events made of five local causally connected clusters anywhere in the detector, while the second one requires at least two clusters on nearby PMT storeys. The selected events are finally kept for online and offline reconstruction as described in [4]. In particular, a fast and robust online algorithm reconstructs tracks in nearly real time [5]. The remaining sample of events is then sent to a more accurate reconstruction algorithm [6] which improves the angular resolution of each event in less than $5 \mathrm{~s}$. Both algorithms use an idealised geometry of the detector that does not take into account the dynamical positioning of the optical modules due to sea current variations. For high-energy tracks, this use limites the angular resolution by about 
$0.1^{\circ}$. These online data are routinely used to look for neutrino counterparts to various astrophysical transient events whose detection is notified directly to ANTARES through both VOEvents ${ }^{1}$ and/or GCN socket and also to send alerts after the detection of interesting neutrino events (as presented in another proceedings [7]). The latest results of these studies are presented hereafter.

\section{Follow-up of gravitational wave events}

The observation of two significant gravitational wave $(\mathrm{GW})$ sources by Advanced LIGO on September $14^{\text {th }}$ and December $26^{\text {th }}, 2015[9,10]$ represents an important step forward in the era of multi-messenger astrophysics.

Since the beginning of the second observing run of Advanced LIGO interferometers on November 30, 2016, ANTARES is receiving gravitational wave alerts in real time. The angular resolution of ANTARES $\left(\sim 0.4^{\circ}\right.$ at $\left.\sim 10 \mathrm{TeV}\right)$ compared to the size of the gravitational wave error box (a few hundreds of square degrees on the sky) offers the possibility to drastically reduce the size of the region of interest in case of a coincident neutrino detection.

About 74 days of LIGO/Hanford and LIGO/Livingston simultaneous science data have been collected up to June 2017. The average reach of the LIGO network for binary merger events is around $70 \mathrm{Mpc}$ for $1.4+1.4 \mathrm{M}_{\odot}, 300 \mathrm{Mpc}$ for $10+10 \mathrm{M}_{\odot}$ and $700 \mathrm{Mpc}$ for $30+30 \mathrm{M}_{\odot}$ mergers, with relative variations in time of the order of $10 \%$. Prior to a commissioning break that occurred in May 2017, 7 triggers, identified by online analysis using a loose false-alarm-rate threshold of one per month, were identified and shared with partner collaborations who have signed memoranda of understanding with LIGO/Virgo for electromagnetic and neutrino follow-up. Each of the 7 alerts were followed by the ANTARES neutrino telescope by searching for a potential neutrino counterpart.

A thorough investigation of the data via an offline GW analysis enabled to confirm a first significant detection which occurred on January 042017 and produced after the merger of two black holes with 31.2 and 19.4 solar masses respectively [11]. We used ANTARES's online reconstruction pipeline [4] which selects up-going neutrino candidates with atmospheric muon contamination less than 10\%. Directional and temporal coincidences between GW170104 and reconstructed HEN candidates were searched for. Relying on the methodology defined in [12], we looked for $(i)$ temporal coincidences within a $\pm 500 \mathrm{~s}$ time window around the GW alert and (ii) spatial overlap between the $90 \%$ probability contour of GW150914 and the neutrino point spread function. No neutrino candidates temporally coincident with GW170104 were found with ANTARES within the $\pm 500 \mathrm{~s}$ time window. The results of the nearly real-time analysis has been transmitted to LIGO/Virgo follow-up community through a GCN (now publicly available [13]) less than 24 hours after the release of the alert ${ }^{2}$. The absence of neutrino candidate both temporally and positionally coincident with GW170104 allowed us to derive a preliminary upper limit on the spectral fluence emitted in neutrinos by the source at $90 \%$ confidence level, as a function of the location of the source in equatorial coordinates and assuming a standard $d N / d E \propto E^{-2}$ spectral model. Figure 1

\footnotetext{
${ }^{1}$ standardized language adopted by the International Virtual Observatory Alliance.

${ }^{2} \mathrm{An}$ automated notification was not generated by LIGO/Virgo after the detection of this event as the calibration of Handford detector's had to be checked beforehand. A first alert was distributed to collaborating astronomers 6 hours after the detection.
} 
(left) shows in each direction of the sky the most stringent fluence upper limit (U.L.) as provided to the LIGO/Virgo follow-up community on January 24, 2017.

Using the constraints on the distance of the GW source and the neutrino fluence U.L., we derived the U.L. on the total energy emitted in neutrinos by this source. This was obtained by integrating the emission between $100 \mathrm{GeV}$ and $100 \mathrm{PeV}$ for each spectral model and each location in the sky map. The total energy U.L. depends on the source distance and equatorial coordinates and is shown in Figure 1 (right) inside the $90 \%$ GW error box.

An optimized offline search for a neutrino counterpart to GW170104 is currently performed in the ANTARES collaboration. Results should be published soon, as it as been done previously for GW150914, GW151226 and LVT151012 [14, 15].
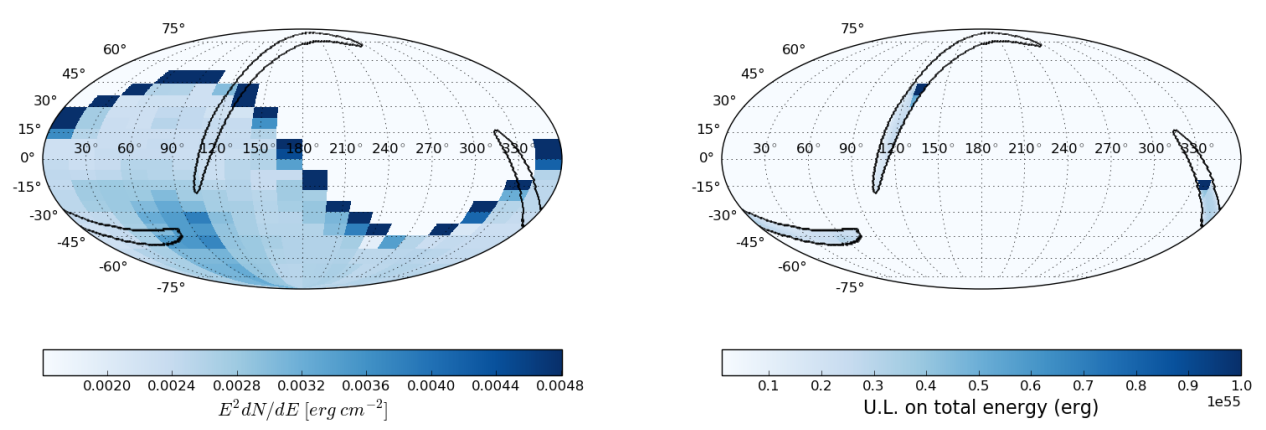

Figure 1: ANTARES $90 \%$ C.L. upper limits on the HEN spectral $v_{\mu}+\bar{v}_{\mu}$ fluence (left) and on the total energy emitted through neutrinos (right) from GW170104 assuming a $d N / d E \propto E^{-2}$ spectral model. Maps in equatorial coordinates. The black contours show the $90 \%$ C.L. contour for the GW skymap.

\section{Follow-up of IceCube HEN events}

IceCube is currently the largest neutrino telescope. Located at the geographic South Pole, it is composed of 86 detection lines distributed over a cubic-kilometer of ice. High-energy events starting into the detector (HESE, see e.g. [1]) and extremely high-energy ones (EHE, with energy above $1 \mathrm{PeV}$ ) are received by the Astrophysical Multi-messenger Observatory Network (AMON, [16]) and distributed to the community via an alert of the $\mathrm{GCN}^{3}$. A coincident detection by both IceCube and ANTARES would be a significant proof of the astrophysical origin of these neutrino candidates and would point directly to the position of the source in the sky. In this context, the ANTARES collaboration is performing a follow-up analysis of each IceCube event whose position is below the horizon of ANTARES (which could consequently yield to an up-going event at the time of the alert). Up to now, ANTARES has followed the four alerts (3 HESE and 1 EHE) in the field-of-view of the telescope (see Figure 2). Two other events occurred in the field-of-view of ANTARES but were retracted by IceCube after further analysis which revealed a background origin. No neutrino candidates were found compatible with one of the alerts within a time window

\footnotetext{
${ }^{3}$ https://gcn.gsfc.nasa.gov/amon.html
} 
up to \pm 1 hour. We used these non-detections to derive preliminary $90 \%$ confidence level upper limits on the radiant neutrino fluence of these events of the order of $\sim 15 \mathrm{GeV} \mathrm{cm}^{-2}$ and $\sim 30 \mathrm{GeV}$ $\mathrm{cm}^{-2}$ for the $\mathrm{E}^{-2}$ and the $\mathrm{E}^{-2.5}$ spectral models respectively (see Table 1). These results have been published as GCN circulars within some hours after the alerts [17, 18, 19, 20].
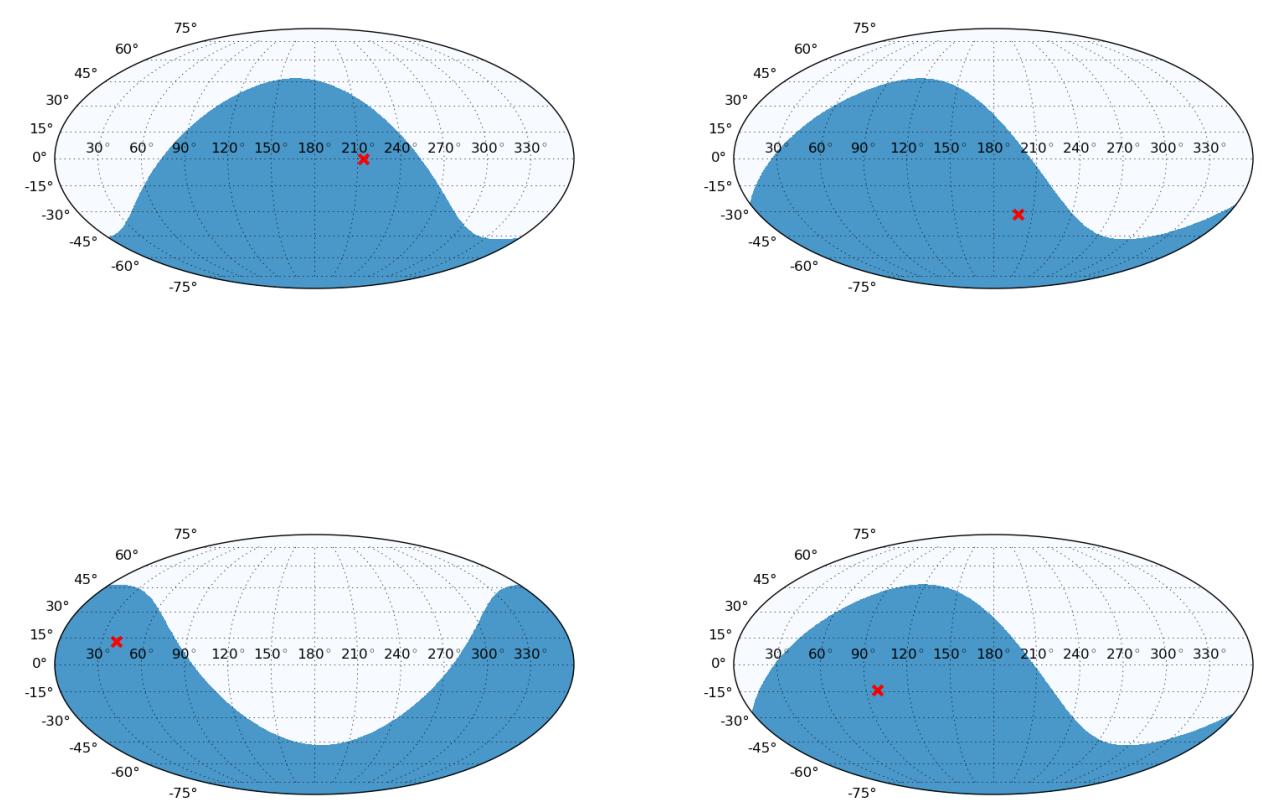

Figure 2: Visibility map of the IceCube alerts (top left: IC160731A, top right: IC160814A, bottom left: IC161103A, bottom right: IC170321A) in equatorial coordinates showing the field-of-view of the ANTARES neutrino telescope at the event time (blue part of the map). The red cross indicates the position of the neutrino candidate observed by IceCube.

\begin{tabular}{|c|c|c|}
\hline IceCube event & \multicolumn{2}{|c|}{ Fluence U.L. $\left(\mathrm{erg} \mathrm{cm}^{-2}\right)$} \\
& $d N / d E \propto E^{-2}$ & $d N / d E \propto E^{-2.5}$ \\
\hline IC160731A (EHE) & $2.210^{-2}$ & $4.310^{-2}$ \\
IC160814A (HESE) & $2.510^{-2}$ & $7.010^{-2}$ \\
IC161103A (HESE) & $2.110^{-2}$ & $5.110^{-2}$ \\
IC170321A (HESE) & $2.610^{-2}$ & $4.210^{-2}$ \\
\hline
\end{tabular}

Table 1: ANTARES fluence upper limits at 90\% C.L. for each IceCube neutrino candidate.

\section{Follow-up of Gamma-Ray Bursts and Fast-Radio Bursts}

Transient astrophysical events are observed all over the electromagnetic spectrum and in particular at both ends of the spectrum, in the radio and gamma-ray domains where we respectively 
observe fast radio bursts (FRBs) and gamma-ray bursts (GRBs). While the latter are probably related to either the collapse of massive stars or the merger of two compact objects, the sources producing the former are still unknown. If hadrons are accelerated in relativistic outflows of both GRBs and FRBs, TeV-PeV neutrinos might be produced by photo-hadronic interactions. Dedicated offline analyses are performed by the ANTARES collaboration (see e.g. [21, 22, 23]) and preliminary results of the more recent ones will be presented at this conference [24, 25]. In particular, a stacked analysis based on the full ANTARES data sample from 2008 to 2016 enables to constrain the contribution of GRBs to the diffuse flux of cosmic neutrinos. On the other hand, looking for individual GRBs and FRBs helps to constrain theoretical models of neutrino emission.

ANTARES is able to react to external alerts sent through GCN after the detection of a GRB. More than 500 GRBs detected by Swift and Fermi have been followed by ANTARES in realtime so far (see Figure 3). Data analysis can be done in two alternative ways. A search for muontrack neutrino counterpart in the standard online dataset described in Section 2 is performed in real-time within $15 \mathrm{~min}$ around the detection and $2^{\circ}$ from the GRB position. To ensure the quality of the data at the alert time, the detector stability is checked over several hours before the alert. The result of the search is sent by email within 15 min after the release of the GCN. In case of a coincident neutrino detection, a dedicated offline analysis is run to confirm the result and compute its significance (expected to be higher than $3 \sigma$ in most of the cases).

Alternatively, a specific data taking mode is activated jointly with the standard one in case of a GRB alert. All raw data covering a preset period (typically 2 min, depending on the background rate, the number of data processing computers, and the size of the RAM) are saved to disk without any filtering [26]. Data buffering in the filter processors enables to store the data up to about one minute before the actual GCN alert. In most cases, it consequently includes data collected by ANTARES before the GRB occurred, which can be used to search for a neutrino signal occurring before the gamma ray emission. These unfiltered data can be analysed with a less strict filtering compared to the standard online filtering and a reconstruction algorithm optimised for energies below $1 \mathrm{TeV}$ [27] can be applied to increase the detection efficiency (by a factor of $\sim 2$ at 100 $\mathrm{GeV}$ and $\sim 30 \%$ at $10 \mathrm{TeV}$ ). A dedicated algorithm searching for time and space correlations in a small region of interest around the GRB position is finally used as in standard offline analyses. An analysis based on this approach has recently been published by the ANTARES collaboration to test the photospheric model of neutrino emission in GRBs [21].

As for FRBs, the Parkes radiotelescope, located in Australia, is the main discovery instrument so far and the SUrvey for Pulsars and Extragalactic Radio Bursts (SUPERB) is underway on this instrument [28]. One of the main obstacles for constraining the nature of the FRBs is the latency between the detection of the burst and the starting of multi-wavelength and multimessenger follow-up observations aimed to identify a FRB counterpart. The SUPERB program was designed to drastically reduce the time needed to send a notification to the community. In this context, ANTARES is receiving alerts issued by the SUPERB collaboration in case of a Fast Radio Burst detection since 2015. The ANTARES collaboration has recently been involved in the multi-wavelength and multi-messenger study of FRB 150215 detected with the Parkes radiotelescope by SUPERB [29]. The datastream was searched for upgoing track events from a point-like source within a time window of up to \pm 1 day around the FRB detection in a region of interest of $2^{\circ}$ 


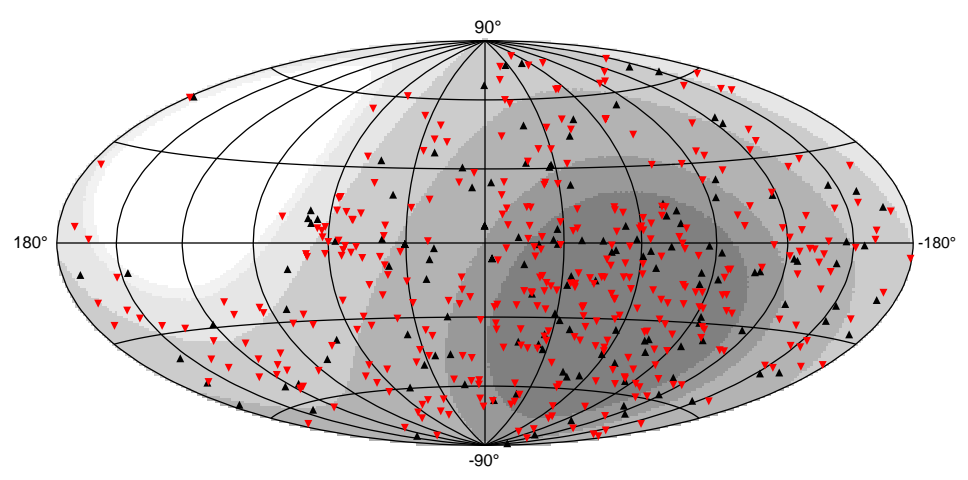

Figure 3: Positions of the the GRBs followed by ANTARES (red ticks indicate those detected by Fermi while black ticks those observed by Swift). The shade of grey indicates the ANTARES visibility. The map is in galactic coordinates.

centered on the position of the Parkes beam center. No neutrinos were detected coincident with this transient event. Consequently, a neutrino radiant fluence ${ }^{4}$ upper limit at $90 \%$ confidence level has been computed together with an upper limit on the total energy emitted in high-energy neutrinos (assuming a redshift of $z=0.56$ as constrained by radio data). Results are given in Table 2 for a spectral model $d N / d E \propto E^{-2}$.

\begin{tabular}{|c|c|}
\hline Fluence U.L. $\left(\mathrm{erg} \mathrm{cm}^{-2}\right)$ & Total energy U.L. $(\mathrm{erg})$ \\
\hline $1.4 \times 10^{-2}$ & $1.4 \times 10^{55}$ \\
\hline
\end{tabular}

Table 2: Results of the FRB150215 neutrino follow-up performed with ANTARES.

\section{Conclusion}

By simultaneously monitoring at least half of the sky, neutrino telescopes are well-suited to detect transient sources. In this context, multi-messenger approaches are destined for a bright future and will help to probe the physical processes at work in these objects. In addition to offline analyses, that search for time and space coincidences of ANTARES events with electromagnetic, gravitational wave or neutrino signals [2, 30,31], ANTARES is also able to handle external alerts in real time (as described in this document) and to trigger electromagnetic follow-up of interesting neutrino candidates [7, 8]. Furthermore, the capability of the ANTARES data acquisition system to store a few minutes of raw data coincident with a GCN alert extends the possibility of increasing offline sensitivity to transient astrophysical events. In the near future, KM3NeT, currently under deployment in the Mediterranean sea, will further develop these dedicated multi-messenger programs that will benefit from an increase of both the discovery potential (by a factor of $\sim 50$ with respect to ANTARES) and the angular resolution of muon tracks and cascade events [32].

\footnotetext{
${ }^{4}$ Defined as $\int E d N / d E d E$.
} 


\section{References}

[1] Aartsen M G et al. (IceCube), ApJ, 809(1), 98 (2015).

[2] Sánchez-Losa A et al. (on behalf of the ANTARES collaboration), these proceedings.

[3] Aguilar J et al., Nucl. Instrum. Meth., A570 107 (2007).

[4] Ageron M et al., Astropart. Phys., 35:530-536 (2012).

[5] Aguilar J A et al., Astropart. Phys., 342011 652-662 (2011).

[6] Adrían-Martinez S et al., ApJ, 760:53 (2012).

[7] Dornic D et al. (on behalf of the ANTARES collaboration), these proceedings.

[8] Adrían-Martínez S et al., (ANTARES), JCAP, 02:62 (2016).

[9] Abbott B P et al., Phys. Rev. Lett., 116061102 (2016).

[10] Abbott B P et al., Phys. Rev. Lett., 116241103 (2016).

[11] Abbott B P et al., Phys. Rev. Lett., 118221101 (2017).

[12] Baret B et al., Astropart. Phys., 35 1:7 (2011).

[13] The circulars exchanged between collaborating astronomers and LIGO/Virgo regarding GW170104 are archived at https://gen.gsfc.nasa.gov/other/G268556.gcn3.

[14] Adrían-Martínez S et al., Phys. Rev. D, 93122010 (2016).

[15] Adrían-Martínez S et al., Phys. Rev. D in press, arXiv:1703.06298 122010 (2017).

[16] Smith M W E et al., Astropart. Phys., 45:56-70 (2013).

[17] Dornic D \& Coleiro A, on behalf of ANTARES, ATeL, 9324 (2016).

[18] Dornic D \& Coleiro A, on behalf of ANTARES, ATeL, 9440 (2016).

[19] Dornic D \& Coleiro A, on behalf of ANTARES, ATeL, 9715 (2016).

[20] Dornic D \& Coleiro A, on behalf of ANTARES, ATeL, 10189 (2017).

[21] Albert A et al., Mon. Not. Roy. Astron. Soc., 469906 (2017).

[22] Adrían-Martinez S et al., Astron. Astrophys., 559 (2013).

[23] Adrían-Martinez S et al., Eur. Phys. J. C, 77:20 (2017).

[24] Celli S (on behalf of the ANTARES collaboration), these proceedings.

[25] Sanguineti M et al. (on behalf of the ANTARES collaboration), these proceedings.

[26] Bouwhuis M (on behalf of the ANTARES collaboration), Procs. ICRC 2009, arXiv:0908.0818 (2009).

[27] Visser E, Doctoral Thesis, Leiden University (2015).

[28] Keane E F et al., arXiv1706.04459, submitted to Mon. Not. Roy. Astron. Soc..

[29] Petroff E et al., Mon. Not. Roy. Astron. Soc., 469, 4465:4482, (2017) .

[30] Pradier T et al. (on behalf of the ANTARES collaboration), these proceedings.

[31] Illuminati $\mathrm{G}$ et al. (on behalf of the ANTARES collaboration), these proceedings.

[32] Adrían-Martínez S et al., JPhG, 438 (2016). 